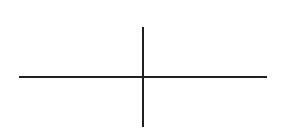

Tohoku Math. J.

57 (2005), 247-260

\title{
CONTACT PAIRS
}

\author{
GiAnluCA BANDE AND AMINE HADJAR
}

(Received August 11, 2003, revised June 18, 2004)

\begin{abstract}
We introduce a new geometric structure on differentiable manifolds. A Contact Pair on a $2 h+2 k+2$-dimensional manifold $M$ is a pair $(\alpha, \eta)$ of Pfaffian forms of constant classes $2 k+1$ and $2 h+1$, respectively, whose characteristic foliations are transverse and complementary and such that $\alpha$ and $\eta$ restrict to contact forms on the leaves of the characteristic foliations of $\eta$ and $\alpha$, respectively. Further differential objects are associated to Contact Pairs: two commuting Reeb vector fields, Legendrian curves on $M$ and two Lie brackets on the set of differentiable functions on $M$. We give a local model and several existence theorems on nilpotent Lie groups, nilmanifolds, bundles over the circle and principal torus bundles.
\end{abstract}

1. Introduction. The aim of this paper is to study some differential Pfaffian forms of constant class. This notion was introduced by E. Cartan (cf. [4], [6]). Global problems relative to constant class forms have been efficiently studied in the case of maximal class: contact forms, symplectic forms and generalized contact forms (cf. [1]).

In fact, we introduce a new geometric structure called Contact Pair. More precisely, a Contact Pair (C.P.) of type $(h, k)$ on a $(2 h+2 k+2)$-dimensional manifold $M$ is a pair of Pfaffian forms $(\alpha, \eta)$ satisfying the following properties:

$$
\begin{array}{cl}
d \alpha^{h+1}=0, & d \eta^{k+1}=0 \text { and } \\
\alpha \wedge d \alpha^{h} \wedge \eta \wedge d \eta^{k} & \text { is a volume form on } M .
\end{array}
$$

The forms $\alpha, \eta$ have constant classes $2 h+1$ and $2 k+1$, respectively.

Some differential objects can be naturally associated to such a structure. The characteristic foliations of $\alpha$ and $\eta$ are transverse and complementary. Their leaves are contact manifolds of dimension $2 k+1$ and $2 h+1$, respectively. We give more general notions of a Reeb vector field and Legendrian curves. We can also associate two Lie brackets on the algebra $\mathcal{C}^{\infty}(M)$. We show that contact pairs of the same type $(h, k)$ admit a local model, like contact and symplectic forms.

Given the richness of this geometry, we are interested in the existence of Contact Pairs. We give several existence theorems for nilpotent Lie groups, nilmanifolds, bundles over the circle, as well as principal torus bundles which showed their utility in contact geometry ([12], [13], [8], [9], [1]). In the bundles $\left(M^{4}, B_{2}, T^{2}\right)$, where the total space and the base are closed orientable manifolds of dimensions 4 and 2, we construct $T^{2}$-invariant contact pairs of type $(1,0)$.

2000 Mathematics Subject Classification. Primary 53D10; Secondary 57R17.

Key words and phrases. Contact geometry, Reeb vector field, complementary foliations, invariant forms. 


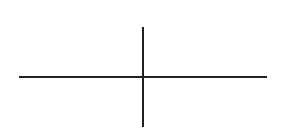

All geometric objects in this paper are supposed $C^{\infty}$. A similar structure called ContactSymplectic Pair is developed in [2] and [3].

The authors would like to express their gratitude to Y. Eliashberg and N. A' Campo for their interest in this work, to M. Bordemann, M. Goze, R. Lutz and M. Zessin for their valuable comments. They kindly acknowledge R. Caddeo, S. Montaldo and P. Piu who made it possible for them to meet.

2. Contact Pairs (C.P.). $\quad$ Let $M$ be a $(2 h+2 k+2)$-dimensional manifold.

Definition 2.1. A pair $(\alpha, \eta)$ of Pfaffian forms on $M$ is said to be a Contact Pair (C.P. for short) of type $(h, k)$ if the following conditions are satisfied:

$$
\begin{aligned}
d \alpha^{h+1}=0, & d \eta^{k+1}=0 \\
\text { and } \alpha \wedge d \alpha^{h} \wedge \eta \wedge d \eta^{k} & \text { is a volume form on } M .
\end{aligned}
$$

Thus the forms $\alpha$ and $\eta$ have constant classes $2 h+1$ and $2 k+1$, respectively. A manifold equipped with a C.P. is clearly orientable.

A C.P. of type $(0,0)$ in a 2-dimensional manifold $M$ is a pair of closed Pfaffian forms with non-vanishing product; if $M$ is closed it follows that $M$ is diffeomorphic to the 2-torus. Therefore we will always suppose $h \geq 1$ or $k \geq 1$.

The simplest example of C.P.s is the following:

"Darboux" C.P.: If $x_{1}, \ldots, x_{2 h+1}, y_{1}, \ldots, y_{2 k+1}$ are coordinate functions on $\boldsymbol{R}^{2 k+2 h+2}$, then the forms

$$
\alpha=d x_{2 h+1}+\sum_{i=1}^{h} x_{2 i-1} d x_{2 i}, \quad \eta=d y_{2 k+1}+\sum_{i=1}^{k} y_{2 i-1} d y_{2 i},
$$

(with the convention: if $h=0$ or $k=0$ the corresponding sum is zero) determine a C.P. of type $(h, k)$ on $\boldsymbol{R}^{2 k+2 h+2}$.

This example is a local model of C.P.'s of type $(h, k)$ (see $\S 3)$.

2.1. Reeb vector fields of a C.P. In this section, we naturally generalize the notion of Reeb vector field classically associated to contact forms.

THEOREM 2.2. Let $(\alpha, \eta)$ be a C.P. of type $(h, k)$ on $M$. Then there exists a unique vector field $X_{\alpha}$ satisfying

$$
\alpha\left(X_{\alpha}\right)=1, \quad i\left(X_{\alpha}\right)\left(d \alpha^{h} \wedge \eta \wedge d \eta^{k}\right)=0
$$

and a unique vector field $X_{\eta}$ satisfying

$$
\eta\left(X_{\eta}\right)=1, \quad i\left(X_{\eta}\right)\left(\alpha \wedge d \alpha^{h} \wedge d \eta^{k}\right)=0 .
$$

ProOF. For the uniqueness, suppose the existence of two vector fields $X_{\alpha}$ and $Y_{\alpha}$ verifying the first two equations. Then the volume form $\alpha \wedge d \alpha^{h} \wedge \eta \wedge d \eta^{k}$ vanishes on $X_{\alpha}-Y_{\alpha}$. Hence $X_{\alpha}=Y_{\alpha}$.

For the existence of $X_{\alpha}$, let us consider the form $\Omega=d \alpha^{h} \wedge \eta \wedge d \eta^{k}$. Its characteristic space is 1 -dimensional at every point, because $\Omega$ is a non-vanishing $(2 k+2 h+1)$-form on 


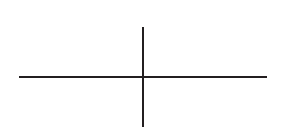

a $(2 k+2 h+2)$-dimensional manifold. Consider a tangent vector $u_{p} \neq 0$ at a point $p$ such that $i\left(u_{p}\right) \Omega_{p}=0$. We set $\left(X_{\alpha}\right)_{p}=u_{p} / \alpha_{p}\left(u_{p}\right)$. This defines a smooth vector field $X_{\alpha}$ on $M$ which satisfies the required conditions.

For the Darboux C.P., the Reeb vector fields are $X_{\alpha}=\partial / \partial x_{2 h+1}$ and $X_{\eta}=\partial / \partial y_{2 k+1}$.

A simple computation shows the following additional properties of Reeb vector fields of a C.P.

Proposition 2.3. The Reeb vector fields $X_{\alpha}, X_{\eta}$ of a C.P. $(\alpha, \eta)$ commute and satisfy the following conditions:

$$
\begin{array}{ll}
\eta\left(X_{\alpha}\right)=0, & i\left(X_{\alpha}\right) d \alpha=i\left(X_{\alpha}\right) d \eta=0, \\
\alpha\left(X_{\eta}\right)=0, & i\left(X_{\eta}\right) d \alpha=i\left(X_{\eta}\right) d \eta=0 .
\end{array}
$$

Hence $X_{\alpha}$ (resp. $X_{\eta}$ ) is tangent to the characteristic foliation of $\eta$ (resp. $\alpha$ ), and coincides on every leaf with the Reeb vector field (in the classical sense) of the contact form induced by $\alpha$ (resp. $\eta$ ) on the leaf.

COROLlary 2.4. A C.P. is invariant by the flows of its Reeb fields.

The following theorem shows that Reeb fields of C.P. have properties similar to Reeb fields of contact forms (cf. [14]):

THEOREM 2.5. The Reeb field $X_{\alpha}$ (resp. $X_{\eta}$ ) of a C.P. $(\alpha, \eta)$ of type $(h, k)$ on $M$ with $h \geqslant 1($ resp. $k \geqslant 1)$ does not admit any closed transverse hypersurface.

Proof. As $h \geqslant 1$, we have $d \alpha^{h} \wedge \eta \wedge d \eta^{k}=d\left(\alpha \wedge d \alpha^{h-1} \wedge \eta \wedge d \eta^{k}\right)$. If there exists a closed transverse hypersurface $S$, then the form

$$
i\left(X_{\alpha}\right)\left(\alpha \wedge d \alpha^{h} \wedge \eta \wedge \eta^{k}\right)=d\left(\alpha \wedge d \alpha^{h-1} \wedge \eta \wedge d \eta^{k}\right)
$$

is an exact volume form on $S$, which is impossible by Stokes' Theorem.

The forms $d \alpha$ and $d \eta$ are absolute integral invariants of $X_{\alpha}$ and $X_{\eta}$. The forms $\alpha$ and $\eta$ are relative integral invariants (cf. [4]).

2.2. Examples of C.P.'s. (1) Let $\left(M_{1}^{2 h+1}, \alpha\right)$ and $\left(M_{2}^{2 k+1}, \eta\right)$ be two contact manifolds and $M=M_{1}^{2 h+1} \times M_{2}^{2 k+1}$. The pair $(\alpha, \eta)$ is a C.P. of type $(h, k)$ on $M$ and it will be called Product C.P. Its Reeb fields are those of the two contact forms considered as vector fields on $M$.

For example, let $\theta_{1}, \theta_{2}, \theta_{3}, \phi$ be coordinate functions on $\boldsymbol{R}^{4}$. Then the pair $\alpha=$ $\sin \theta_{3} d \theta_{1}-\cos \theta_{3} d \theta_{2}, \eta=d \phi$ is a C.P. of type $(1,0)$ on the torus $T^{4}$ and its Reeb fields are $X_{\alpha}=\sin \theta_{3} \partial / \partial \theta_{1}-\cos \theta_{3} \partial / \partial \theta_{2}, X_{\eta}=\partial / \partial \phi$.

(2) Let $M_{1}^{2 h+2}$ be a manifold with a C.P. $(\alpha, \eta)$ of type $(h, 0)$ and $M_{2}^{2 k}$ an open manifold with a volume form $(d \theta)^{k}$, where $\theta$ is a Pfaffian form. The pair $(\alpha, \eta+\theta)$ is a C.P. of type $(h, k)$ on $M_{1}^{2 h+2} \times M_{2}^{2 k}$.

2.3. Characteristic foliations of $\alpha$ and $\eta$. Let $(\alpha, \eta)$ be a C.P. of type $(h, k)$ on $M$. We can naturally associate to it the distribution of vectors on which $\alpha$ and $d \alpha$ vanish, and the 


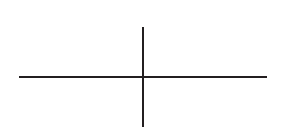

one of vectors on which $\eta$ and $d \eta$ vanish. These distributions are involutive because $\alpha$ and $\eta$ have constant classes. They determine the characteristic foliations of $\alpha$ and $\eta$, noted $\mathcal{F}$ and $\mathcal{G}$, respectively.

The foliations $\mathcal{F}$ and $\mathcal{G}$ are of codimension $2 h+1$ and $2 k+1$, respectively, and their leaves are contact manifolds. This justifies the name of the structure. Moreover, $\mathcal{F}$ and $\mathcal{G}$ are transverse and complementary.

3. Local model of contact pairs. To construct a local model for C.P., we use the existence of characteristic foliations just described. One can easily show the following (see [2]).

THEOREM 3.1. Let $(\alpha, \eta)$ be a C.P. of type $(h, k)$ on $M$, with $h \geqslant 1$. For every point $p$ of $M$, there exists an open neighborhood $V$ of $p$ and a coordinate system on $V$ such that the pair $(\alpha, \eta)$ can be written:

$$
\alpha_{V}=d x_{2 h+1}+\sum_{i=1}^{h} x_{2 i-1} d x_{2 i}, \quad \eta_{V}=d y_{2 k+1}+\sum_{i=1}^{k} y_{2 i-1} d y_{2 i},
$$

with the convention: $\eta_{V}=d y_{1}$ if $k=0$.

Thus every C.P. is locally a product C.P. The open set $V$ will be called a Darboux neighborhood.

4. Further differential objects associated to a C.P. Let $(\alpha, \eta)$ be a C.P. of type $(h, k)$ on a manifold $M, \mathcal{F}$ and $\mathcal{G}$ the characteristic foliations of $\alpha$ and $\eta$, respectively. We can naturally associate to it the following differential objects:

4.1. Characteristic foliations of $d \alpha$ and $d \eta$. Since $d \alpha$ and $d \eta$ have constant classes $2 h$ and $2 k$, they determine two characteristic foliations $\mathcal{F}^{\prime}$ and $\mathcal{G}^{\prime}$ of codimension $2 h$ and $2 k$, respectively.

Each leaf of $\mathcal{F}^{\prime}$ (resp. $\mathcal{G}^{\prime}$ ) is a union of leaves of $\mathcal{F}$ (resp. $\mathcal{G}$ ). Furthermore, it is clear that the pair induced by $(\alpha, \eta)$ on a leaf $F$ of $\mathcal{F}^{\prime}$ (resp. $\mathcal{G}^{\prime}$ ) is a C.P. of type $(0, k)$ (resp. $\left.(h, 0)\right)$ on $F$. These foliations also have the following interesting properties:

Proposition 4.1. Suppose that the characteristic foliation $\mathcal{F}^{\prime}$ of $d \alpha$ (respectively $\mathcal{G}^{\prime}$ of $d \eta$ ) has a closed leaf $F$. Then all the leaves of $\mathcal{F}$ (resp. $\mathcal{G}$ ) lying in $F$ are diffeomorphic, and $F$ fibers over the circle.

Proof. The form $\alpha$ induces on $F$ a non-zero closed Pfaffian form $\alpha_{F}$. Then if $F$ is closed, it fibers over the circle (cf. [15]) and the characteristic leaves of $\alpha_{F}$ are diffeomorphic. But these leaves are exactly those of $\mathcal{F}$ lying in $F$.

4.2. Lie brackets on $\mathcal{C}^{\infty}(M)$ associated to a C.P. Using the contact forms induced on the leaves of $\mathcal{F}$ and $\mathcal{G}$, the algebra $\mathcal{C}^{\infty}(M)$ can be endowed with a pair of Lie brackets. Precisely, to every function $f$ on $M$, we can associate two vector fields $X_{f, \alpha}$ and $X_{f, \eta}$ as follows: 


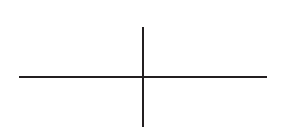

CONTACT PAIRS

On each leaf $G$ of $\mathcal{G}$, there exists a unique vector field $X$ tangent to $G$ such that:

$$
\alpha_{G}(X)=f_{\mid G} \quad \text { and } \quad\left(L_{X} \alpha_{G}\right) \wedge \alpha_{G}=0,
$$

where $\alpha_{G}$ is the contact form induced by $\alpha$ on $G$ (cf. [11]).

The vector field we obtained on $M$ is well defined, smooth and will be noted $X_{f, \alpha}$. In a similar way, we construct $X_{f, \eta}$. Now we can introduce the two Lie brackets.

Definition 4.2. The Lie bracket of $f, g \in \mathcal{C}^{\infty}(M)$ along $\alpha$ is the function:

$$
\{f, g\}_{\alpha}=\alpha\left(\left[X_{f, \alpha}, X_{g, \alpha}\right]\right)
$$

and the Lie bracket along $\eta$ is the function

$$
\{f, g\}_{\eta}=\eta\left(\left[X_{f, \eta}, X_{g, \eta}\right]\right) .
$$

The usual properties of Lie brackets hold.

4.3. Legendrian curves. They are defined as follows:

Definition 4.3. A Legendrian curve of the C.P. $(\alpha, \eta)$ with respect to $\alpha$ is a piecewise differentiable curve $\gamma_{\alpha}$ on $M$ such that

$$
\alpha\left(\dot{\gamma}_{\alpha}\right)=0 \text { and } i\left(\dot{\gamma}_{\alpha}\right)\left(\eta \wedge d \eta^{k}\right) \neq 0 \text { everywhere. }
$$

Similarly, we define a Legendrian curve with respect to $\eta$. The curves must be tangent to $\mathcal{F}$ (resp. $\mathcal{G}$ ), but transverse to $\mathcal{G}$ (resp. $\mathcal{F}$ ). They can join the points as in connected contact manifolds (see [1]):

Proposition 4.4. Any two points on a connected manifold $M$ equipped with a C.P. $(\alpha, \eta)$ can be joined by a Legendrian curve with respect to $\alpha$ and by a Legendrian curve with respect to $\eta$.

5. Topological obstructions. Let $(\alpha, \eta)$ be a C.P. of type $(h, k)$ on a manifold $M$.

Proposition 5.1. If $M$ is a closed manifold, then $H^{2 h+1}(M, \boldsymbol{R}) \neq 0$ and $H^{2 k+1}(M, \boldsymbol{R}) \neq 0$.

Proof. If $H^{2 h+1}(M, \boldsymbol{R})=0$ or $H^{2 k+1}(M, \boldsymbol{R})=0$, the volume form $\alpha \wedge d \alpha^{h} \wedge \eta \wedge d \eta^{k}$ is exact, which is impossible when $M$ is closed.

An immediate consequence (which also follows from the existence of a non-vanishing vector field on a C.P. manifold) is the following:

COROLlaRY 5.2. There is no C.P. on even-dimensional spheres.

By using [15], we have the following result:

Proposition 5.3. If $M$ is closed and equipped with a C.P. of type $(h, 0)$, then $M$ fibers over the circle.

Here are some properties concerning Reeb vector fields: 


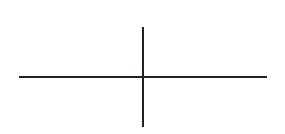

PROPOSITION 5.4. The Reeb vector fields of a C.P. determine a locally free action of $\boldsymbol{R}^{2}$. Every closed orbit is a 2-torus.

Proof. As Reeb vector fields commute, they generate a locally free action of $\boldsymbol{R}^{2}$. Every orbit admits two non-vanishing (Reeb) fields and then its Euler-Poincaré characteristic vanishes.

This action will be called the Reeb action.

THEOREM 5.5. The Reeb action does not admit a closed transversal submanifold of codimension 2.

Proof. If $h=k=0$, it is obvious. Suppose $h \geq 1$ and let $N$ be a 2-codimensional closed transversal submanifold. Then

$$
i(Y) i(X)\left(\alpha \wedge d \alpha^{h} \wedge \eta \wedge d \eta^{k}\right)=d \alpha^{h} \wedge d \eta^{k}=d\left(\alpha \wedge d \alpha^{h-1} \wedge d \eta^{k}\right)
$$

induces an exact volume form on $N$, which is impossible by Stokes' Theorem.

REMARK 5.6. If every orbit of the Reeb action is a closed manifold, then we have a locally free action of the torus. If this action generates a principal fibre bundle, the C.P. (which is invariant) has an empty singular set (see $§ 8$ for details).

6. C.P.'s on nilpotent Lie groups and nilmanifolds. Nilpotent Lie groups and nilmanifolds provide further interesting examples of C.P.'s. Below we present some examples of constructions in dimensions 4 and 6 which can possibly be extended to higher dimensions. We use the classification of nilpotent Lie algebras of dimensions 4 and 6 in [7].

6.1. C.P.'s on nilpotent Lie groups. In order to describe the Lie algebra of a Lie group, we give only the non-zero ordered brackets of the fundamental fields $X_{i}$. Their dual forms will be noted $\omega_{i}$.

EXAMPLE 6.1. Consider the 4-dimensional Lie algebra $n_{4}^{1}$ given by

$$
\left[X_{1}, X_{4}\right]=X_{3}, \quad\left[X_{1}, X_{3}\right]=X_{2} .
$$

The pair $\left(\omega^{2}, \omega^{4}\right)$ determines a C.P. of type $(1,0)$ on the corresponding Lie group.

EXAMPLE 6.2. On the 6-dimensional Lie algebra $n_{6}^{12}$ given by

$$
\left[X_{1}, X_{6}\right]=X_{5}, \quad\left[X_{1}, X_{5}\right]=X_{4}, \quad\left[X_{2}, X_{3}\right]=X_{4},
$$

the pair $\left(\omega^{4}, \omega^{6}\right)$ determines a C.P. of type $(2,0)$ on the corresponding Lie group.

EXAMPLE 6.3. On the group corresponding to the 6-dimensional Lie algebra $n_{6}^{13}$ given by

$$
\left[X_{1}, X_{6}\right]=X_{5}, \quad\left[X_{1}, X_{5}\right]=X_{4}, \quad\left[X_{1}, X_{4}\right]=X_{3}, \quad\left[X_{5}, X_{6}\right]=X_{2},
$$

the pair $\left(\omega^{2}, \omega^{3}\right)$ determines a C.P. of type $(1,1)$.

6.2. C.P.'s on nilmanifolds. We remark that in the previous examples the Lie algebras are rational; thus the unique connected and simply connected Lie groups corresponding to 


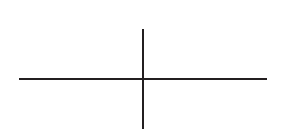

them admit cocompact discontinuous subgroups (cf. [7]). Then the nilmanifolds, obtained as quotients by these subgroups, are closed manifolds equipped with C.P.'s of the same type.

7. Existence theorems of C.P.'s of type $(h, 0)$. As we have seen in $\$ 5.3$, a closed manifold equipped with a C.P. of type $(h, 0)$ fibers over the circle. By using Feldbau's theorem (cf. [5]), we construct non-product C.P.'s $(\alpha, \eta)$ of type $(h, 0)$ on manifolds that fiber over the circle, in such a way that the characteristic foliation of $\eta$ coincides with the bundle foliation. We recall Feldbau's theorem:

Equivalence classes of differentiable fiber bundles over the circle with closed, connected fiber $M$ and structural group $\operatorname{Diff}^{+}(M)$ are in one-to-one correspondence with $\pi_{0}\left(\mathrm{Diff}^{+}(M)\right)$.

If $f \in \operatorname{Diff}^{+}(M)$, the bundle is obtained as the quotient of $\left.M \times\right]-\varepsilon, 1+\varepsilon[, \varepsilon>0$ by the equivalence relation which identifies the points $(x, t) \in M \times]-\varepsilon, \varepsilon$ [ with $h(x, t)=$ $(f(x), 1+t) \in M \times] 1-\varepsilon, 1+\varepsilon\left[\right.$. The total space will be denoted $M_{f}$ and $h$ will be called the gluing diffeomorphism.

THEOREM 7.1. Let $\left(B_{2 h+1}, \omega\right)$ be a connected, closed contact manifold. If $f \in$ $\operatorname{Diff}^{+}\left(B_{2 h+1}\right)$ and $f^{*} \omega=\omega$, then there exists a C.P. $(\tilde{\omega}, \eta)$ of type $(h, 0)$ on $\left(B_{2 h+1}\right)_{f}$. Moreover the pair can be chosen in such a way that every contact leaf of the characteristic foliation of $\eta$ is a contact embedding of $\left(B_{2 h+1}, \omega\right)$.

Proof. Let $\left(B_{2 h+1}, \omega\right)$ be a connected, closed contact manifold, $f \in \operatorname{Diff}^{+}(B)$ such that $f^{*} \omega=\omega$. Consider $\varepsilon>0$ and $\left.I_{\varepsilon}=\right]-\varepsilon, 1+\varepsilon$ [. Let $p_{1}, p_{2}$ be the projections of $B \times I_{\varepsilon}$ on $B$ and $I_{\varepsilon}$, respectively, and $d t$ the canonical volume form of $I_{\varepsilon}$. The pair $\left(p_{1}^{*}(\omega), p_{2}^{*}(d t)\right)$ is a product C.P. of type $(h, 0)$ on $B \times I_{\varepsilon}$, invariant by the gluing diffeomorphism. Thus it induces a C.P. $(\tilde{\omega}, \eta)$ of type $(h, 0)$ on $B_{f}$. Let $\pi: B_{f} \rightarrow S^{1}$ be the canonical projection, $d \theta$ the form on $S^{1}$ induced by $d t$. By construction, we have $\eta=\pi^{*} d \theta$ and its characteristic foliation coincides with the one defined by $\pi$. Let $F=\pi^{-1}(\tau)$ be any fiber and $t \in I_{\varepsilon}$ a representative modulo 1 of $\tau$. As a contactomorphism between $(B, \omega)$ and $\left(F, \tilde{\omega}_{F}\right)$, one can take the one which sends a point $p \in B$ to $(p, t) \in B \times I_{\varepsilon}$ modulo the gluing diffeomorphism $h$.

We shall say that the C.P. $(\tilde{\omega}, \eta)$ constructed above is induced by $\omega$ and $f$. It is a product C.P. if and only if $f$ is isotopic to the identity $\operatorname{map~id}_{B}$.

REMARK 7.2. This theorem gives a method to construct non-product C.P.'s on a bundle over the circle where the fiber $B$ is endowed with a contact form $\omega$ and a diffeomorphism $f$ leaving $\omega$ invariant. But not all C.P.'s are obtained in this way, as the following example shows.

Indeed, consider the forms

$$
\omega=\cos \theta_{3} d \theta_{1}+\sin \theta_{3} d \theta_{2} \quad \text { and } \quad \eta=d \theta_{4}+\lambda d \theta_{1}
$$

on $T^{4}$, where $\lambda$ is an irrational number chosen sufficiently small to ensure the pair $(\omega, \eta)$ to be a C.P. and $\eta$ irrational. Therefore, the characteristic leaves of $\eta$ are open. Thus they cannot be the compact fibers of a bundle over the circle with total space $T^{4}$. 


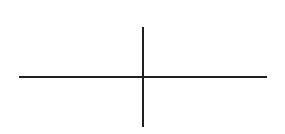

REMARK 7.3. If the form $\eta$ is irrational it is close to a rational form $\beta$. Then if $(\omega, \eta)$ is a C.P. of type $(h, 0)$, so is $(\omega, \beta)$. This shows that any C.P. of type $(h, 0)$ is close to a C.P. as in the previous theorem.

7.1. Non-product examples. Here are some fundamental examples of pairs $(\omega, f)$ on a manifold $B_{2 h+1}$ which give C.P.'s on the associated Feldbau's bundle $\left(B_{2 h+1}\right)_{f}$.

(1) On the torus $T^{3}$, for each integer $n \neq 0$, we consider the contact form $\omega_{n}=$ $\cos \left(n \theta_{3}\right) d \theta_{1}+\sin \left(n \theta_{3}\right) d \theta_{2}$ and the diffeomorphism $f_{n}\left(\theta_{1}, \theta_{2}, \theta_{3}\right)=\left(\theta_{2}, \theta_{1},(\pi / 2 n)-\theta_{3}\right)$.

(2) We can also consider the contact form $\omega=\cos \theta_{3} d \theta_{1}+\sin \theta_{3} d \theta_{2}$ and the diffeomorphism $f\left(\theta_{1}, \theta_{2}, \theta_{3}\right)=\left(\theta_{1},-\theta_{2},-\theta_{3}\right)$ on $T^{3}$.

(3) On the torus $T^{5}$, we have the contact form (see [13])

$$
\begin{aligned}
\omega= & \sin \theta_{2} \cos \theta_{2} d \theta_{1}-\sin \theta_{1} \cos \theta_{1} d \theta_{2} \\
& +\cos \theta_{1} \cos \theta_{2} d \theta_{3}+\left(\sin \theta_{1} \cos \theta_{3}-\sin \theta_{2} \sin \theta_{3}\right) d \theta_{4} \\
& +\left(\sin \theta_{1} \sin \theta_{3}+\sin \theta_{2} \cos \theta_{3}\right) d \theta_{5}
\end{aligned}
$$

and the diffeomorphism

$$
f\left(\theta_{1}, \theta_{2}, \theta_{3}, \theta_{4}, \theta_{5}\right)=\left(\pi-\theta_{1},-\theta_{2}, \frac{\pi}{2}-\theta_{3}, \theta_{5}, \theta_{4}\right) .
$$

(4) Let $U^{*} M$ be the unit cotangent bundle of an $n$-dimensional Riemannian manifold $(M, g), \alpha$ the Liouville contact form on $U^{*} M$. If $f \in \operatorname{Diff}^{+}(M)$ has finite order $p$, we choose the isomorphism $F$ of the bundle $U^{*} M$ defined by

$$
F\left(x, \eta_{x}\right)=\left(f(x),\left(f^{-1}\right)^{*}\left(\eta_{x}\right) /\left\|\left(f^{-1}\right)^{*}\left(\eta_{x}\right)\right\|_{g}\right)
$$

for each $x \in M$ and $\eta_{x} \in U_{x}^{*} M$. Clearly, its order is $p$ and $F^{*} \alpha=\lambda \alpha$ where $\lambda\left(x, \eta_{x}\right)=$ $1 /\left\|\left(f^{-1}\right)^{*}\left(\eta_{x}\right)\right\|_{g}$. Hence $\omega=\sum_{k=1}^{p}\left(F^{k}\right)^{*} \alpha$ is a contact form on $U^{*} M$ which satisfies $F^{*} \omega=\omega$. The C.P. we obtain in $\left(U^{*} M\right)_{F}$ is of type $(n-1,0)$.

The diffeomorphisms considered in (1), (2) and (3) do not induce the identity map on the first homotopy group of the manifold. Hence, they are not isotopic to id. In (4), if $f$ is chosen non-isotopic to id, so is $F$. In this way we obtain non-product C.P.'s.

7.2. Constructions on $\left(M_{3}\right)_{f}$ where $M_{3}$ is the total space of a principal $S^{1}$-bundle. Let $B_{2}$ be a closed, connected, orientable surface of genus $g \geq 2$. First, we construct a family $D\left(B_{2}\right)$ of orientation preserving diffeomorphisms of $B_{2}$ which are of finite order and not isotopic to the identity $\operatorname{map} \mathrm{id}_{B}$. Next, we intend to lift these diffeomorphisms $f$ to certain principal $S^{1}$-bundles $M_{3}$ over $B_{2}$, as isomorphisms $\tilde{f}$ of the bundle (also of finite order). Finally, we construct $S^{1}$-invariant contact forms $\omega$ on $M_{3}$ satisfying $\tilde{f}^{*} \omega=\omega$. Therefore, we will have non-product C.P. of type $(1,0)$ on each bundle $\left(M_{3}\right) \tilde{f}$.

7.2.1. The family $D\left(B_{2}\right)$. Let $\Delta(g)=\{2\} \cup\{m \in N, m \geq 2, m \mid(g-1)\}$ and $n \in$ $\Delta(g)$. We embed $B_{2}$ in $\boldsymbol{R}^{3}$ and give diffeomorphisms $\varphi_{f, n}$ of order $n$ which are not isotopic to $\mathrm{id}_{B}$, as follows:

First case $(n \mid(g-1)$ with $g \geq 3)$ : Let $l=(g-1) / n$. We consider a 2-torus $T$ of revolution. Let $C_{1}$ be a meridian circle of $T$. By iterating $n$ times the $(2 \pi / n)$-rotation around 


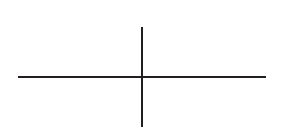

CONTACT PAIRS

the revolution axis, the images of $C_{1}$ are $n$ new circles $C_{i}$ (except $C_{n+1}=C_{1}$ ). We glue $l$ handles on one of the $n$ connected components $T_{i}$ of $T-\bigcup_{i} C_{i}$ (after removing $2 l$ disks). By each iteration of the same rotation, we will have $l$ new handles glued on $T$. Let $S_{n}^{g}$ be the surface so obtained and $P_{n}=\left\{f: B_{2} \rightarrow S_{n}^{g} \mid f\right.$ is a diffeomorphism $\}$. For each $f \in P_{n}$, this rotation induces a orientation preserving diffeomorphism $\varphi_{f, n}$ on $B_{2}$ of order $n$.

Second case ( $n=2$ with $g$ even): Consider the unit sphere $S$. Let $C_{1}$ be a circle containing the poles, $l=g / n$, and $T_{i}$ the two connected components of $S-C_{1}$. We glue $l$ handles on $T_{1}$ after removing $2 l$ disks, and by the $\pi$-rotation sending $T_{1}$ to $T_{2}$, we have $l$ new handles on $T_{2}$. As in the first case, we obtain a surface $S_{2}^{g}$ and we put $P_{2}=\{f$ : $B_{2} \rightarrow S_{2}^{g} \mid f$ is a diffeomorphism $\}$. The surface $S_{2}^{g}$ is also invariant by the above symmetry, which therefore induces for every $f \in P_{2}$ a diffeomorphism $\varphi_{f, 2}$ of order 2 on $B_{2}$. We put $C_{2}=\varphi_{2}\left(C_{1}\right)=C_{1}$ where $\varphi_{2}$ is the $\pi$-rotation.

By construction, for each $f \in P_{n} B_{2}=f^{-1}\left(\bigcup_{i} \Sigma_{i}\right)$ where $\Sigma_{i}$ are $n$ 2-dimensional compact connected submanifolds with boundary $C_{i} \cup C_{i+1}$, and interior $T_{i}$ with the $l$ handles. For each $i$ and $f, f^{-1} \circ \varphi_{f, n} \circ f\left(\Sigma_{i}\right)=\Sigma_{i+1}$ and $\Sigma_{n+1}=\Sigma_{1}$.

We set $D\left(B_{2}\right)=\left\{\varphi_{f, n} \mid n \in \Delta(g), f \in P_{n}\right\}$.

7.2.2. How to lift these diffeomorphisms? In the trivial bundle $\left(B_{2} \times S^{1}, B_{2}, S^{1}\right)$, we can evidently lift any diffeomorphism $f$ of $B_{2}$ by $\tilde{f}(x, \theta)=(f(x), \theta)$. However, it is possible to find non-trivial principal $S^{1}$-bundles $\left(M_{3}, B_{2}, S^{1}\right)$ on which the elements of $D\left(B_{2}\right)$ can be lifted as isomorphisms (of finite order) of the bundle. For example, since $B_{2}$ was considered as a Riemannian submanifold of $\boldsymbol{R}^{3}$, the elements $f$ of $D\left(B_{2}\right)$ are isometries (see 7.2.1). So, their tangent maps $\tilde{f}$ induced on the unit tangent bundle $U B_{2}$ are isomorphisms. We can also consider for each integer $k \neq 0$ the bundle $\left(E_{(k)}, B_{2}, S^{1}\right)$ associated to $\left(U B_{2}, B_{2}, S^{1}\right)$ with total space $E_{(k)}=U B_{2} \times S^{1} S^{1}$ quotient of $U B_{2} \times S^{1}$ by the $S^{1}$-action $\left(z,\left(p, z^{\prime}\right)\right) \rightarrow$ $\left(p z, z^{-k} z^{\prime}\right)$ (see [10, p. 54]). This bundle is still a principal $S^{1}$-bundle. Every element $f \in$ $D\left(B_{2}\right)$ can be lifted as follows: $\hat{f}_{k}([p, z])=[\tilde{f}(p), z]$ for each representative $(p, z) \in U B_{2} \times$ $S^{1}$ of an equivalence class $[p, z]$ in $E_{(k)}$. For each $f \in D\left(B_{2}\right)$, the orientation preserving isomorphisms $\tilde{f}$ and $\hat{f}_{k}$ have the same order as $f$, and are not isotopic to the identity map.

7.2.3. Construction of contact forms on $M_{3}$. Let $M_{3}\left(B_{2}, S^{1}, q\right)$ be a principal $S^{1}$ bundle where the total space $M_{3}$ is a closed, connected and orientable 3-manifold. Let $f$ be any element of $D\left(B_{2}\right)$. Assume that $F$ is a orientation preserving isomorphism of the bundle with finite order inducing $f$ on the base. Then we have:

THEOREM 7.4. There exists an $S^{1}$-invariant contact form $\omega$ on $M_{3}$ such that $F^{*} \omega=$ $\omega$.

PROOF. In the above notation, let $n \in \Delta(g)$ and $f=\varphi_{n}$.

First case $(n \mid(g-1)$ with $g \geq 3)$ : Let $\eta_{1}$ be a germ of an $S^{1}$-invariant contact form along the torus $q^{-1}\left(C_{1}\right)$. Next, we put $\eta_{2}=\left(F^{-1}\right)^{*} \eta_{1}$. Then we have a germ of an $S^{1}$ invariant contact form along $\partial\left(q^{-1}\left(\Sigma_{1}\right)\right)$. According to [8, Lemma 1.3 and Theorem 3.3], this extends to an $S^{1}$-invariant contact form $\omega_{1}$ on $q^{-1}\left(\Sigma_{1}\right)$. Let $\omega$ be the form on $M_{3}$ whose 


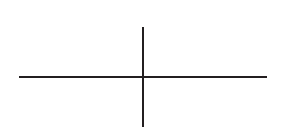

restriction to each $q^{-1}\left(\Sigma_{i}\right)$ is $\omega_{i}=\left(F^{-i+1}\right)^{*} \omega_{1}$. It is well defined, $S^{1}$-invariant and satisfies $F^{*} \omega=\omega$.

Second case ( $n=2$ with g even): First, let us construct a germ $\eta$ of an $S^{1}$-invariant contact form along the torus $q^{-1}\left(C_{1}\right)$ such that $F^{*} \eta=\eta$. There exists a tubular neighborhood $U$ of this torus isomorphic to $\left.V=T^{2} \times\right]-1,1[$, such that $F$ induces the isomorphism $F_{V}:\left(\theta_{1}, \theta_{2}, t\right) \rightarrow\left(\theta_{1}, \pi-\theta_{2},-t\right)$ of $V$. The $S^{1}$-invariant contact form $\tilde{\eta}=d \theta_{1}+t d \theta_{2}$ on $V$ satisfies $F_{V}^{*} \tilde{\eta}=\tilde{\eta}$. Hence we have $\eta$. This germ also extends to an $S^{1}$-invariant contact form $\omega_{1}$ on $q^{-1}\left(\Sigma_{1}\right)$. Set $\omega_{2}=\left(F^{-1}\right)^{*} \omega_{1}$ on $q^{-1}\left(\Sigma_{2}\right)$, to get a global contact form on $M_{3}$ which coincides with $\omega_{i}$ on $q^{-1}\left(\Sigma_{i}\right)$ for $i=1,2$.

Thus $\left(M_{3}\right)_{F}$ is endowed with a C.P. of type $(1,0)$.

8. Existence of C.P.'s of type $(1,0)$ on principal torus bundles $\left(M^{4}, B_{2}, T^{2}\right)$. Several existence problems for contact forms have been solved using an additional invariance condition under which the space carries geometrically useful structures (see [12], [13], [8], [9], [1]). We proceed along the same lines. Let us consider principal torus bundles $\left(M^{4}, B_{2}, T^{2}, \pi\right)$ where the base and the total space are connected, closed and orientable.

Let $\theta=\sum_{i=1}^{2} \theta^{i} \otimes e_{i}$ be a connection form, $\Omega=\sum_{i=1}^{2} \Omega^{i} \otimes e_{i}$ its curvature form and $X_{1}, X_{2}$ the fundamental vector fields generated by $e_{1}, e_{2}$.

Let $(\alpha, \eta)$ be a pair of Pfaffian forms on $M^{4}$. These forms are $T^{2}$-invariant if and only if they can be written as follows:

$$
\begin{gathered}
\alpha=\pi^{*}(\beta)+\pi^{*}\left(f_{1}\right) \theta^{1}+\pi^{*}\left(f_{2}\right) \theta^{2}, \\
\eta=\pi^{*}(\gamma)+\pi^{*}\left(g_{1}\right) \theta^{1}+\pi^{*}\left(g_{2}\right) \theta^{2},
\end{gathered}
$$

where $\beta$ and $\gamma$ are Pfaffian forms and $f_{i}, g_{i}$ functions on the base space.

The pair $(\alpha, \eta)$ is an invariant C.P. of type $(1,0)$ if and only if the following three conditions are satisfied on $B_{2}$ :

CONDITION 8.1.

$$
\begin{gathered}
\beta \wedge\left(g_{2} d f_{1}-g_{1} d f_{2}\right)+\left(g_{2} f_{1}-g_{1} f_{2}\right) \cdot\left(d \beta+f_{1} \Omega^{1}+f_{2} \Omega^{2}\right) \\
+\left(f_{2} d f_{1}-f_{1} d f_{2}\right) \wedge \gamma \neq 0 \quad \text { everywhere on } B_{2}
\end{gathered}
$$

CONDition 8.2.

$$
d f_{1} \wedge d f_{2}=0
$$

CONDITION 8.3.

$$
\begin{gathered}
d g_{1}=d g_{2}=0 \quad \text { (thus } g_{1} \text { and } g_{2} \text { are constants), } \\
\text { and } \quad d \gamma+g_{1} \Omega^{1}+g_{2} \Omega^{2}=0 .
\end{gathered}
$$

We call the singular set of $(\alpha, \eta)$ the set $S$ on which the function

$$
(\alpha \wedge \eta)\left(X_{1}, X_{2}\right)=\pi^{*}\left(g_{2} f_{1}-g_{1} f_{2}\right)
$$




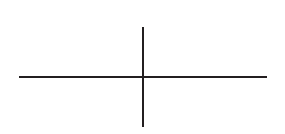

vanishes or equivalently $S=\pi^{-1}(\Sigma)$, where $\Sigma$ is the set of zeros of the function $h=$ $g_{2} f_{1}-g_{1} f_{2}$. Geometrically, $S$ is the set of points of $M^{4}$ where the forms $\alpha$ and $\eta$ induced on the fibers $T^{2}$ are proportional.

8.1. Nature of the singular set. If $\Sigma$ is not empty and does not coincide with $B_{2}$, one of the constants $g_{1}, g_{2}$ is not zero. We suppose $g_{2} \neq 0$, and by the condition 8.1, we have

$$
\left(g_{2} \beta-f_{2} \gamma\right) \wedge d h+g_{2} h \cdot\left(d \beta+f_{1} \Omega^{1}+f_{2} \Omega^{2}-d f_{2} \wedge \gamma\right) \neq 0 .
$$

Where $h$ vanishes, we have $\left(g_{2} \beta-f_{2} \gamma\right) \wedge d h \neq 0$, which implies that $\Sigma$ is a closed orientable embedded submanifold of $B_{2}$ of codimension 1 , and therefore a finite disjoint union of circles.

The set $\Sigma$, when $\Sigma \neq \emptyset$ and $\Sigma \neq B_{2}$, verifies the following obvious property:

CONDITION 8.4. To every connected component of $B_{2}-\Sigma$ we can attach a sign + or - (the sign of the function $h$ ), in such a way that two adjacent components have opposite signs.

If $S=\pi^{-1}(\Sigma)$ is the singular set of an invariant C.P., $\Sigma$ satisfies necessarily one of the three following properties:

(1) $\Sigma$ coincides with $B_{2}$,

(2) $\Sigma$ is empty,

(3) $\quad \Sigma$ is a 1-codimensional closed orientable embedded submanifold of $B_{2}$ satisfying the condition 8.4.

We shall see in the next paragraph that each of these cases is possible.

8.2. Existence theorems on $\left(M^{4}, B_{2}, T^{2}\right)$. We shall show that for every set $\Sigma$ in each one of the three cases of the previous paragraph there exists an invariant C.P. $(\alpha, \eta)$ having $\pi^{-1}(\Sigma)$ as singular set.

8.2.1. The case $\Sigma=B_{2}$. The base space is necessarily the 2-torus. In fact:

THEOREM 8.5. Let $\left(M^{4}, B_{2}, T^{2}\right)$ be a principal torus bundle where total and base space are closed, connected and orientable. There exists an invariant C.P. of type $(1,0)$ with singular set $M^{4}$ if and only if $B_{2}$ is the 2-torus.

Proof. Suppose the existence of such a C.P. on $M^{4}$. Then we have $\beta, \gamma, f_{i}, g_{i}$ on $B_{2}$ satisfying the conditions $8.1,8.2,8.3$ and $h \equiv 0$. These conditions become

(1) $\left(f_{2} d f_{1}-f_{1} d f_{2}\right) \wedge \gamma \neq 0$,

(2) $d f_{1} \wedge d f_{2}=0$,

(3) $g_{1}=g_{2}=0$ and $d \gamma=0$.

This implies that $\gamma$ is a non-singular closed Pfaffian form. Thus $B_{2}$ is a torus.

Conversely, if the base space is a torus with "pseudo-coordinates" $\theta^{1}$ and $\theta^{2}$, we can choose $\gamma=d \theta^{1}, f_{1}=\sin \theta^{2}, f_{2}=\cos \theta^{2}$ to get an invariant C.P. with singular set $M^{4}$.

8.2.2. The case $\Sigma$ empty. There is a constraint on the bundle. In fact: 


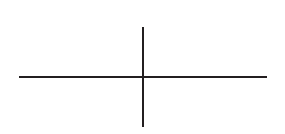

THEOREM 8.6. Let $\left(M^{4}, B_{2}, T^{2}\right)$ be a principal torus bundle with total and base space closed, connected and orientable. There exists an invariant C.P. of type $(1,0)$ with empty singular set if and only if the characteristic classes of the bundle do not vanish simultaneously.

Hence in the trivial bundle $\left(T^{2} \times T^{2}, T^{2}, T^{2}\right)$, there is no such C.P.

Proof. Suppose that there exists an invariant C.P. $(\alpha, \beta)$ with empty singular set. In the notation above, at least one of $g_{1}, g_{2}$ is not zero. If $g_{2} \neq 0$, we have $f_{1}=\left(g_{1} f_{2}+h\right) / g_{2}$. Condition 8.1 becomes

$$
g_{2} h^{2} d(\beta / h)+g_{2} h \cdot\left(f_{1} \Omega^{1}+f_{2} \Omega^{2}\right)+\left(f_{2} d h-h d f_{2}\right) \wedge \gamma \neq 0,
$$

which gives

$$
g_{2} h \cdot d(\beta / h)-h \cdot d\left(\left(f_{2} / h\right) \cdot \gamma\right)+g_{2} \cdot\left(f_{1} \Omega^{1}+f_{2} \Omega^{2}\right)+f_{2} d \gamma \neq 0 .
$$

Since $d \gamma=-g_{1} \Omega^{1}-g_{2} \Omega^{2}$, we have

$$
d\left(g_{2} \beta / h\right)-d\left(\left(f_{2} / h\right) \cdot \gamma\right)+\Omega^{1} \neq 0 \text { everywhere. }
$$

This condition implies that $\int \Omega^{1} \neq 0$ and thus $\left[\Omega^{1}\right] \neq 0$.

Conversely, suppose that there is a non-vanishing characteristic class (for example $\left[\Omega^{1}\right] \neq$ $0)$ and consider the pair

$$
\begin{aligned}
\alpha & =\pi^{*}(\beta)+\pi^{*}\left(g_{2}\right) \theta^{1}+\pi^{*}\left(-g_{1}\right) \theta^{2}, \\
\eta & =\pi^{*}(\gamma)+\pi^{*}\left(g_{1}\right) \theta^{1}+\pi^{*}\left(g_{2}\right) \theta^{2},
\end{aligned}
$$

where $g_{1}, g_{2}$ are constants and $\beta, \gamma$ Pfaffian forms on $B_{2}$. This determines a C.P. of type $(1,0)$ with empty singular set if and only if

$$
\begin{aligned}
d \gamma+g_{1} \Omega^{1}+g_{2} \Omega^{2}=0 & \text { and } \\
\left(g_{2}^{2}+g_{1}^{2}\right) \cdot\left(d \beta+g_{2} \Omega^{1}-g_{1} \Omega^{2}\right) \neq 0 & \text { everywhere. }
\end{aligned}
$$

As $\int \Omega^{1} \neq 0$ we can find $\left(g_{1}, g_{2}\right) \neq(0,0)$ such that $g_{1} \int \Omega^{1}+g_{2} \int \Omega^{2}=0$. Thus $l=$ $g_{2} \int \Omega^{1}-g_{1} \int \Omega^{2} \neq 0$, and there exists a form $\gamma$ satisfying $d \gamma+g_{1} \Omega^{1}+g_{2} \Omega^{2}=0$. Now choose a volume form $\Phi$ whose integral is 1 . Then we have $\int l \Phi=g_{2} \int \Omega^{1}-g_{1} \int \Omega^{2}$, which implies the existence of some $\beta$ satisfying $d \beta+g_{2} \Omega^{1}-g_{1} \Omega^{2} \neq 0$ everywhere.

8.2.3. $\Sigma$ is a submanifold of codimension 1 . There is no obstruction on the bundle and we have:

THEOREM 8.7. Consider a principal torus bundle $\left(M^{4}, B_{2}, T^{2}, \pi\right)$ with closed, connected, orientable total and base space. Let $\Sigma$ be a closed orientable 1-codimensional imbedded submanifold of $B_{2}$, satisfying the condition 8.4. There exists an invariant C.P. $(\alpha, \eta)$ of type $(1,0)$ on $M^{4}$ having $\pi^{-1}(\Sigma)$ as the singular set.

An example is given in Remark 7.2 where the singular set is the union of two 3-tori.

Before proving the theorem, we give a technical lemma which will be useful in the following constructions (see [12, p. 5] for a proof). 


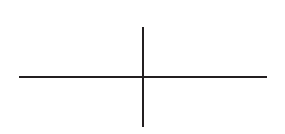

LEMMA 8.8. Le B be a closed orientable 2-dimensional manifold and $\Sigma$ a 1-codimensional compact orientable embedded submanifold satisfying the condition 8.4. Then there exists a function $h$ and a Pfaffian form $\beta$ on $B$ such that $\Sigma=h^{-1}(0)$ and the form $h \cdot d \beta+\beta \wedge d h$ is a volume form on $B$.

Proof. (of Theorem 8.7) We have to find $\beta, \gamma, f_{i}, g_{i}$ such that the conditions 8.1, 8.2 and 8.3 are satisfied.

First step: $\quad$ By Lemma 8.8, there exist $h$ and $\beta_{0}$ such that

$$
\beta_{0} \wedge d h+h \cdot d \beta_{0} \neq 0, \quad h^{-1}(0)=\Sigma .
$$

Second step: We construct constants $g_{i}$ and a form $\gamma$ satisfying the condition 8.3. If the characteristic classes $\left[\Omega^{i}\right]$ vanish, then $\Omega^{1}=d \gamma_{1}, \Omega^{2}=d \gamma_{2}$. We take $\left(g_{1}, g_{2}\right) \neq(0,0)$ arbitrarily and $\gamma=-\left(g_{1} \gamma_{1}+g_{2} \gamma_{2}\right)$.

If there is a non-vanishing characteristic class, we can always find $\left(g_{1}, g_{2}\right) \neq(0,0)$ such that: $g_{1} \int \Omega^{1}+g_{2} \int \Omega^{2}=0$. This implies that there exists $\gamma$ satisfying $d \gamma+g_{1} \Omega^{1}+g_{2} \Omega^{2}=$ 0 .

Third step: We construct the functions $f_{i}$. We set:

$$
f=h /\left(k_{1} g_{2}-k_{2} g_{1}\right), \quad f_{1}=k_{1} f, \quad f_{2}=k_{2} f,
$$

where $k_{i}$ are constants such that $k_{1} g_{2}-k_{2} g_{1} \neq 0$. Then the condition 8.2 is satisfied.

Last step: We set $\beta=r \beta_{0}$ where $r$ is a real number sufficiently large such that the condition 8.1 is satisfied.

REMARK 8.9. By Remark 7.2, if in the previous three theorems $\eta$ is irrational then the C.P. $(\alpha, \eta)$ cannot be obtained as in Theorem 7.1.

\section{REFERENCES}

[ 1] G. BAnde, On generalized contact forms, Differential Geom. Appl. 11 (1999), 257-263.

[2] G. BAnde, Formes de contact généralisé, couples de contact et couples contacto-symplectiques, Thèse de Doctorat, Université de Haute Alsace, 2000.

[ 3 ] G. BAnde, Couples contacto-symplectiques, Trans. Amer. Math. Soc. 355 (2003), 1699-1711.

[ 4 ] E. Cartan, Leçons sur les invariants intégraux, Hermann, Paris, 1922.

[ 5 ] J. Feldbau, Sur la classification des espaces fibrés, C. R. Acad. Sci. Paris, 208, 1936.

[6] C. Godbillon, Géométrie différentielle et mécanique analytique, Hermann, Paris, 1969.

[ 7 ] M. Goze And Y. Khakimdjanov, Nilpotent Lie algebras, Math. Appl. 361, Kluwer Acad. Publ., Dordrecht, 1996.

[ 8 ] A. Hadjar, Sur un problème d'existence relatif de formes de contact invariantes en dimension trois, Ann. Inst. Fourier (Grenoble) 42 (1992), 891-904.

[ 9 ] A. HAdJAR, Sur les structures de contact régulières en dimension trois, Trans. Amer. Math. Soc. 347 (1995), 2473-2480.

[10] S. Kobayashi And K. NomizU, Foundations of differential geometry I, Interscience Publishers, a division of John Wiley \& Sons, New York-London, 1963.

[11] P. Libermann And C. M. Marle, Géométrie symplectique, bases théoriques de la mécanique, Publ. Math. Univ. Paris VII, vol. I, II, III and IV, 1986. 
[12] R. LuTZ, Structures de contact sur les fibrés en cercles en dimension trois, Ann. Inst. Fourier (Grenoble) 27 (1977), 1-15.

[13] R. LuTZ, Sur la géométrie des structures de contact invariantes, Ann. Inst. Fourier (Grenoble) 29 (1979), 283-306.

[14] G. REEB, Sur certaines propriétés topologiques des trajectoires des systèmes dynamiques, Acad. Roy. Belgique Cl. Sci. Mém. Coll. in $8^{\circ}, 27,1952$.

[15] D. TischLER, On fibering certain foliated manifolds over $S^{1}$, Topology 9 (1970), 153-154.

UNIVERSITÀ DI CAGLIARI

VIA OSPEDALE 72

09129 CAGLIARI

ITALIA

E-mail address: gbande@unica.it
Université de Haute Alsace

4 RUE DES FRÈRES LUMIÈRE

68093 Mulhouse Cedex

FRANCE

E-mail address: A.Hadjar@uha.fr 